\title{
Submatrix inversion approach to the ab initio Green's function method for electrical transport *
}

\author{
Tomofumi Tada ${ }^{\dagger}$ and Satoshi Watanabe \\ Department of Materials Engineering, Graduate School of Engineering, \\ The University of Tokyo, 7-3-1 Hongo, Bunkyo-ku, Tokyo 113-8656, Japan, and \\ CREST, Japan Science and Technology Agency, 4-1-8 Honcho Kawaguchi, Saitama 332-0012, Japan
}

(Received 14 November 2005; Accepted 11 March 2006; Published 3 May 2006)

\begin{abstract}
In order to theoretically examine electrical transport, including the effects of semi-core orbitals, we propose a submatrix inversion approach within the framework of Green's function method. The efficiency and reliability of the proposed approach is investigated for a model system of a $\mathrm{Au}_{6}$ linear chain between $\mathrm{Au}(001)$ surfaces using the BPW91 functional and a modified LANL2MB basis set. For the energy integration of Green's function, the large integral window ranging from $-116 \mathrm{eV}$ to $E_{F}(=-5.0 \mathrm{eV})$ is successfully converted into three small windows [-116 eV, $-106 \mathrm{eV}],[-67 \mathrm{eV},-55 \mathrm{eV}]$, and $\left[-15 \mathrm{eV}, E_{F}\right]$. The computation time of the submatrix inversion approach is faster by about $40 \%$ than that for the full-matrix inversion. At the same time, excellent agreement of the computational results between the submatrix and full-matrix inversion is achieved. Further, we propose a new quantity that is useful to determine an appropriate submatrix size. [DOI: 10.1380/ejssnt.2006.484]
\end{abstract}

Keywords: Density functional calculations; Green's function methods; Electrical transport; Gold; Nanowires

\section{INTRODUCTION}

Recently, various tools and techniques such as scanning tunneling microscopy (STM), atomic force microscopy $(\mathrm{AFM})$, and the break junction technique have enabled the manipulation of materials at the atomic scale. They yield, for example, nanosized junctions consisting of atomic or molecular wires, which have been extensively investigated as building blocks of nano-devices. For atomic wires, measurements of the electrical conductance have been successfully performed so far: gold atomic wires, for instance, show a conductance of about $1.0 G_{0}[1-3]$, where $G_{0}$ is the quantum unit of conductance $2 e^{2} / h$. This result is supported by many theoretical calculations based on the density functional theory (DFT) which lead to an understanding of the transport mechanism [4-7]. On the contrary, for electrical conductance measurements of molecular wires, many problems remain to be clarified, though several measurements and calculations have been carried out. One of the significant problems is a large discrepancy between measured and calculated conductances (e.g., the benzene dithiolate system [4, 8-13]). There are many possible origins of this discrepancy. In experimental measurements, it is difficult to determine the number of molecules attached to electrodes and the sites on which molecules are adsorbed [8, 9]. In theoretical studies on the other hand, the models adopted for computations are often too simplistic when compared to experimental situations. The validity of DFT for electrical transport may also be questionable in some cases [13].

Stimulated by the above situation, many groups have been creating and improving computational methods and programs to examine electrical transport through molecular wires [14-17]. We have also carried out studies [1821], adopting the Green's function method and incorporating the method with the ab initio program GAUSSIAN

*This paper was presented at International Symposium on Surface Science and Nanotechnology (ISSS-4), Saitama, Japan, 14-17 November, 2005.

${ }^{\dagger}$ Corresponding author: tada@cello.t.u-tokyo.ac.jp
[22]. The use of GAUSSIAN may have advantages, since the program has a sophisticated implementation of DFT. It also includes implementation of methods beyond DFT such as time-dependent DFT and configuration interactions.

Several groups have already studied electrical transport using GAUSSIAN with the Green's function method $[4,5]$. They adopted the DFT method, and considered nano-contact systems composed of gold electrodes and a single molecule for their investigations. However, the use of GAUSSIAN is often inefficient to study electrical transport of nano-contact systems including heavy atoms. Since the basis sets with effective core potentials for heavy atoms supported in GAUSSIAN involve semi-core states which are located far below the Fermi level (for example, the orbital energies of $5 s$ and $5 p$ states of gold in LANL2MB or LANL2DZ [23-25] are about -111 and -61 $\mathrm{eV}$, respectively), the energy integration of Green's function requires heavy computation. To overcome this difficulty, in the present work, we use Green's function in terms of molecular orbitals to find a reasonable contour path for energy integration, and we propose the submatrix inversion approach to reduce the computation time, especially for the semi-core or core states. In addition, the efficiency and reliability of the proposed approach are confirmed for a model system of a $\mathrm{Au}_{6}$ linear chain between $\mathrm{Au}$ surfaces. In the following sections, we use the term core states for $5 s$ and $5 p$ states of gold for convenience.

\section{METHOD}

\section{A. Green's function in terms of molecular orbitals}

Figure 1 shows the model system composed of $\mathrm{Au}(001)$ surfaces and $\mathrm{a} \mathrm{Au}_{6}$ linear chain. For the investigation of electrical transport through two electrodes, the system is typically divided into three regions; the left electrode, the scattering region, and the right electrode. According to Green's function method for electrical transport [26], the transport property of the system is represented in terms of Green's function of the scattering region, which is written 


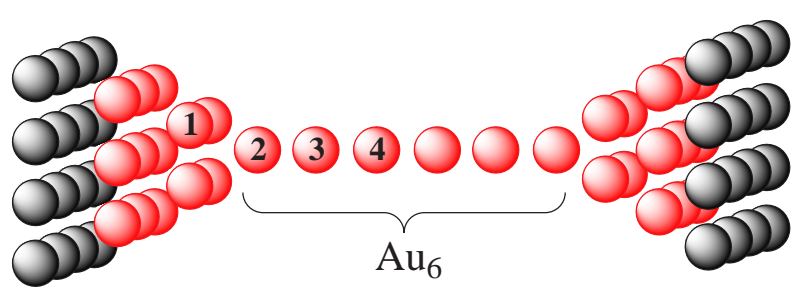

FIG. 1: $\mathrm{Au}_{64}$ cluster including an $\mathrm{Au}_{6}$ linear chain. The atoms included in the scattering region are depicted as red and the atoms within the electrodes are black. The distance between neighboring atoms is fixed to be $2.88 \AA$. The electronic populations of the numbered atoms are analyzed in the latter part of this study.

as

$$
\mathbf{G}(E)=\left[E \mathbf{S}-\mathbf{F}-\Sigma_{\mathrm{L}}-\Sigma_{\mathrm{R}}\right]^{-1},
$$

where $\mathbf{S}$ and $\mathbf{F}$ are the overlap and Fock matrices of the scattering region, respectively $[4,5]$. The couplings between the scattering region and electrodes are taken into account through the self energy $\Sigma$. The self energy is represented in terms of Green's function of the electrodes and non-diagonal elements of $E \mathbf{S}-\mathbf{F}$ between the scattering region and electrodes. To obtain Green's function of electrodes, we adopted the surface Green's function method (decimation technique) [27]. In this study, the matrices required to calculate Green's function of the scattering region were obtained with the BPW91 functional [28, 29] and a modified LANL2MB basis set. The modification of the basis set was done to eliminate the most diffusive atomic orbital $(\mathrm{AO})$ from the original LANL2MB since the diffusive $\mathrm{AO}$ causes difficulty in numerical integration of Green's function. Although the basis set modification involves an important physical meaning, the final conclusions reported in this study are not affected by the modification. Thus, we do not describe the details of the modified LANL2MB basis set.

When the system is in an equilibrium state, the density matrix of the scattering region can be calculated as

$$
\rho=-\frac{1}{\pi} \operatorname{Im} \int_{-\infty}^{E_{F}} \mathbf{G}(E) d E .
$$

On the other hand, if the system is in a nonequilibrium state, we must use the nonequilibrium Green's function instead of the equilibrium Green's function [26]. However, the following approach for core states is not significantly affected by the difference between the two states, and we thus focus our attention on the equilibrium Green's function method.

In the energy integral of Eq. (2), a complex contour is very useful because the number of energy points for the integration is significantly reduced compared to the number of energy points using the path along the real axis [30]. We can see this situation from Fig. 2 in which the imaginary part of Green's function $(-1 / \pi \operatorname{Tr}[\operatorname{Im} \mathbf{G}])$ is shown on the complex energy plane. If we choose the red path for the energy integration, we need many energy points (i.e., a fine mesh) since the variations of the imaginary part of Green's function along the real axis are very large. The large variations along the real axis are caused due to the

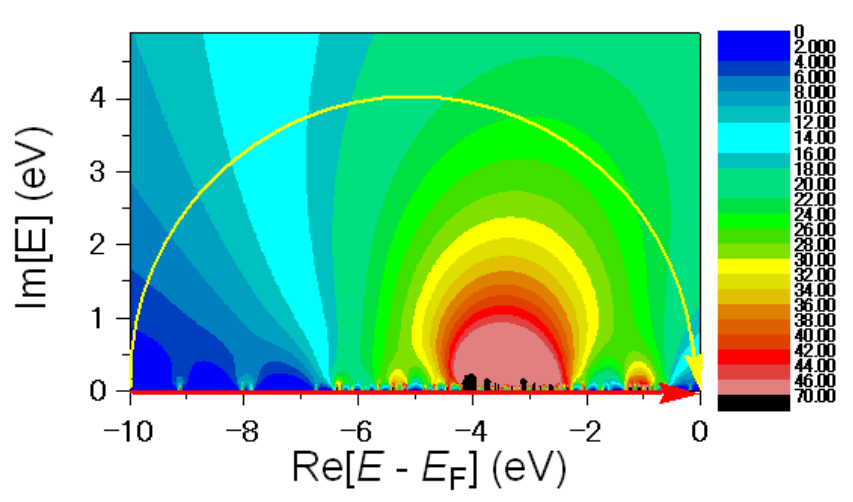

FIG. 2: Complex energy plane mapping of the imaginary part of Green's function for the system shown in Fig. 1. The color bar shows the values of the imaginary part of Green's function on the complex energy plane. The red line is the integral path along the real axis and the yellow one is a contour path. The poles of Green's function are concentrated in the region from -1 to $-6 \mathrm{eV}$ below the Fermi level.

fact that Green's function of Eq. (1) has poles just below the real axis (the lower-half complex energy plane). On the other hand, the yellow contour will reduce the number of energy points because the variations in the imaginary part of Green's function are very small along the complex contour. This is a significant advantage of the Green's function method.

When we only have to consider valence states, the complex contour shown in Fig. 2 is adequate. However, this is not the case when we have to consider core states explicitly. For example, let us consider the complex contour for gold systems including core states. When a typical basis set with effective core potentials such as LANL2MB or LANL2DZ is adopted, $5 s, 5 p, 5 d, 6 s$, and $6 p$ AOs are explicitly taken into account in the calculations for gold. Since $5 s$ AO is located at $-111 \mathrm{eV}$, we have to integrate Green's function in the energy window of $\left[-116 \mathrm{eV}, E_{F}\right.$ $(=-5.0 \mathrm{eV})]$ (Fig. 3(A)). This integration will however be an extremely laborious task since the energy range of the integration is very large. To avoid this difficulty, the division of the contour path as shown in Fig. 3(B) is useful and practical. The effective energy windows in the divided contour are $[-116 \mathrm{eV},-106 \mathrm{eV}],[-67 \mathrm{eV},-55 \mathrm{eV}]$, and [-15 $\left.\mathrm{eV}, E_{F}\right]$. Note that the divided contour must contain all poles of Green's function, otherwise the computed electron density using Eq. (2) will significantly deviate from the neutral state of the system, leading to incorrect results not consistent with the associated physics.

Here, the question arises as to how the divided contour can be obtained. If every value of Green's function on the complex energy plane can be calculated over the whole region of $\left[-116 \mathrm{eV}, E_{F}\right]$ (i.e., the mapping of Green's function), we will obtain a reliable divided contour because the positions of poles in Green's function are a good measure to construct the divided contour. Such a computation, however, is essentially impractical because of the heavy computation required using infinitely large numbers of energy points. Hence, we convert Green's function in terms of AOs to the one in terms of molecular orbitals (MOs). This enables us to construct the divided contour without mapping Green's function on the complex energy 


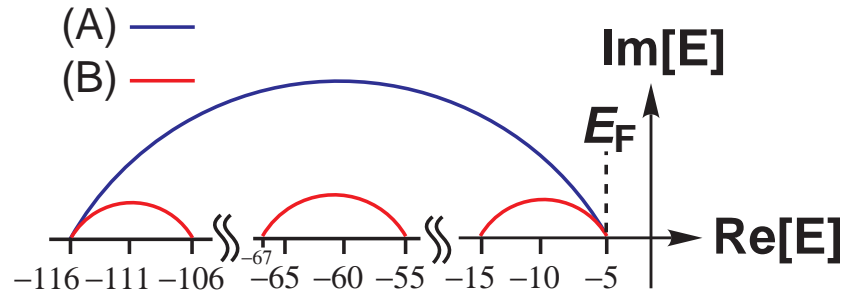

FIG. 3: (A) Non-divided (blue) and (B) divided (red) contours in the complex energy plane. The electronic states for $5 s$ and $5 p$ states are computed using the contours for $[-116 \mathrm{eV},-106$ $\mathrm{eV}]$ and $[-67 \mathrm{eV},-55 \mathrm{eV}]$, respectively.

plane, such as shown in Fig. 2. Transformation using an eigenvector $\mathbf{C}$ which satisfies $\mathbf{F C}=\mathbf{S C E}$ leads to Green's function in terms of MOs $\tilde{\mathbf{G}}(E)$ as

$\mathbf{G}(E)=\mathbf{C}\left[\frac{1}{\mathbf{C}^{+}\left[E \mathbf{S}-\mathbf{F}-\Sigma_{\mathrm{L}}-\Sigma_{\mathrm{R}}\right] \mathbf{C}}\right] \mathbf{C}^{+}=\mathbf{C} \tilde{\mathbf{G}}(E) \mathbf{C}^{+}$

Since vector $\mathbf{C}$ does not depend on energy $E$, we can write the energy integral of Eq. (2) as

$$
\rho=-\frac{1}{\pi} \operatorname{Im} \mathbf{C}\left[\int_{-\infty}^{E_{F}} \tilde{\mathbf{G}}(E) d E\right] \mathbf{C}^{+} .
$$

Using the relation $\mathbf{C}^{+} \mathbf{S C}=\mathbf{1}, \tilde{\mathbf{G}}(E)$ can be represented as

$$
\tilde{\mathbf{G}}(E)=\left[E \mathbf{1}-\tilde{\mathbf{F}}-\tilde{\Sigma}_{\mathrm{L}}-\tilde{\Sigma}_{\mathrm{R}}\right]^{-1},
$$

where $\tilde{\mathbf{F}}$ and $\tilde{\Sigma}$ are the Fock and self-energy matrices in terms of MOs, respectively. Since $\tilde{\mathbf{F}}$ is already diagonal, the eigenvalues of $\tilde{\mathbf{F}}$ are a good guide for the estimation of the positions of poles in Green's function. In addition, the real and imaginary parts of the diagonal elements of $\tilde{\Sigma}$ indicate level-shifting and level-broadening of each eigenlevel respectively. We therefore construct the divided contour from these eigenvalues of $\tilde{\mathbf{F}}$ and diagonal elements of $\tilde{\Sigma}$, as shown in Fig. 3 (B).

\section{B. Submatrix inversion approach}

As mentioned above, $\tilde{\mathbf{F}}$ in Eq. (5) is a diagonal matrix, and thus we can readily distinguish between core and valence states from the values of the diagonal elements. When the energy separation between core and valence states is very large, a submatrix in $[E \mathbf{1}-\tilde{\mathbf{F}}-\tilde{\Sigma}]$ is sufficient to calculate Green's function in Eq. (5). Here, we have dropped the indices L and R of $\tilde{\Sigma}$ for simplicity. For example, when we just focus on the core states ( $5 s$ and $5 p$ states), the submatrix including only the core states in $\tilde{\mathbf{F}}$ and the corresponding self-energy submatrix are used to obtain Green's function for the core states. The division of the full-matrix in Eq. (5) into submatrices is defined as

$$
[E \mathbf{1}-\tilde{\mathbf{F}}-\tilde{\Sigma}]^{-1}=\left(\begin{array}{c|c}
E \mathbf{1}-\tilde{\mathbf{F}}_{c}-\tilde{\Sigma}_{c} & -\tilde{\Sigma}_{c v} \\
\hline-\Sigma_{v c} & E \mathbf{1}-\tilde{\mathbf{F}}_{v}-\Sigma_{v}
\end{array}\right)^{-1}
$$

where indices $c$ and $v$ mean core and valence, respectively. That is, the submatrix we have to consider for the core states is $\left[E \mathbf{1}-\tilde{\mathbf{F}}_{c}-\tilde{\Sigma}_{c}\right]$. Assuming that the interactions between the core and valence states (i.e., $\tilde{\Sigma}_{c v}$ and $\tilde{\Sigma}_{v c}$ ) are negligible, we can invert this submatrix separately. This approach reduces the computation time for the matrix inversion in Eq. (5).

Since the reliability of this approach depends on how small the interactions between the core and valence states are, the division into $c$ and $v$ shown in Eq. (6) may lead to large numerical errors in the case that the interactions between the core and valence states are large. However, it is possible to avoid this difficulty by extending the size of the submatrix in the manner proposed below.

First, we have to examine whether the perturbation caused by the valence states is large. One may think that the matrix elements of self energy $\tilde{\Sigma}_{c v}$ are useful to determine the magnitude of the interactions between the core and valence states. However, we found that the matrix elements cannot be a good measure since some of the elements of $\tilde{\Sigma}_{c v}$ are often large even when the division in Eq. (6) is successful. We thus have to investigate the perturbation of Green's function caused by the valence states in a straightforward way. The perturbation to the self energy of the core states from the valence states can be written as

$$
\Sigma_{\mathrm{v}}^{\prime}=\tilde{\Sigma}_{\mathrm{cv}}\left[\mathrm{E} \mathbf{1}-\tilde{\mathbf{F}}_{\mathrm{v}}-\tilde{\Sigma}_{\mathrm{v}}\right]^{-1} \tilde{\Sigma}_{\mathrm{vc}}
$$

Note that the matrix inversion $\left[E \mathbf{1}-\tilde{\mathbf{F}}_{c}-\tilde{\Sigma}_{c}-\Sigma_{\mathrm{v}}^{\prime}\right]^{-1}$ includes interactions from the valence state perfectly, and thus yields results which are the same as computed from the full-matrix in Eq. (6). On the other hand, the computed Green's function of the core states $\tilde{\mathbf{G}}_{c}(\equiv[E \mathbf{1}-$ $\left.\tilde{\mathbf{F}}_{c}-\tilde{\Sigma}_{c}\right]^{-1}$ ) can provide the perturbation effect within the Born approximation as $\tilde{\mathbf{G}}_{c} \Sigma_{\mathrm{v}}^{\prime} \tilde{\mathbf{G}}_{\mathrm{c}}$. Considering the fact that the imaginary part of Green's function corresponds to the density matrix (see Eq. (2)), we define a quantity $\rho^{\text {Born }}$, which represents the perturbation of the density matrix, as

$$
\rho^{B o r n}(E)=-\frac{1}{\pi} \operatorname{Tr}\left[\operatorname{Im} \tilde{\mathbf{G}}_{c}(E) \Sigma_{\mathrm{v}}^{\prime}(\mathrm{E}) \tilde{\mathbf{G}}_{\mathrm{c}}(\mathrm{E})\right] .
$$

As we show in the next section, $\rho^{\text {Born }}$ turns out to be a good measure for the valid division into $c$ and $v$ in Eq. (6). 


\section{RESULTS AND DISCUSSION}

The typical steps in Green's function method to investigate transport properties are as follows. (1) Selfconsistent field (SCF) calculation using electron density computed from Green's function to determine the electronic states, and (2) calculation of the transmission function using the converged Green's function after the first step [26]. Although the final target of this study is the calculation of transport properties, we focused our attention on the first step to confirm the reliability of the submatrix inversion approach. We thus calculated the Mülliken populations for the gold system shown in Fig. 1. To compare our results with the correct results, we also calculated the Mülliken populations from the full-matrix of Green's function in Eq. (6), which we call full-matrix inversion. In this study, the Mülliken populations obtained from the first iteration of the SCF procedure in Green's function method are presented. That is, the matrix inversion with $\mathbf{S}$ and $\mathbf{F}$ obtained for the $\mathrm{Au}_{64}$ cluster (Fig. 1) using GAUSSIAN is performed to calculate the Mülliken populations. If the efficiency and reliability of the present approach cannot be confirmed even in the first iteration of SCF, this approach cannot be applied to the calculation including the SCF procedure. In addition, results without the effects of $\mathrm{SCF}$ are suitable to analyze the properties of the present approach. Hence the results from the first iteration of SCF are analyzed as the first step.

Table I shows the computed Mülliken populations of gold atoms and cpu times in the energy window of [-116 $\mathrm{eV},-106 \mathrm{eV}$, which corresponds to the region of the $5 \mathrm{~s}$ states. We considered five sizes of the submatrix; the matrix sizes of Submatrices (A), (B), (C), (D), and (E) are $32,90,128,158$, and 190, respectively. Since the scattering region is composed of 32 gold atoms, the submatrix, including 32 poles from the $5 s$ states, is at least required. This minimum submatrix is Submatrix (A), while Submatrix (B) includes $5 p$ states located below $-60.4 \mathrm{eV}$, together with the $5 s$ states. The $5 s$ and $5 p$ states are completely included in Submatrix (C), and valence states located below -11.0 and $-9.5 \mathrm{eV}$ are added further in Submatrices (D) and (E), respectively. The size of the fullmatrix is 448. As we anticipated in the previous section, the computation time of the submatrix inversion is faster by about $40 \%$ than that for the full-matrix inversion as shown in Table I. In addition, the submatrix inversions of (A), (B), and (C) show excellent agreement with the fullmatrix inversion. This result means that the interactions between the core $(5 s$ and $5 p)$ and valence $(5 d, 6 s$, and $6 p)$ are small. On the other hand, submatrix inversions (D) and (E) cannot provide reasonable results especially for $\mathrm{Au}(1)$. This is despite the fact that these submatrices include more states (i.e., valence states). The largest difference in (D) is about 0.1 e. This discrepancy indicates that if the inclusion of valence states into the core submatrix is performed in a wrong manner (i.e., using a wrong balance of inclusion), numerical errors will become large. This is the difficulty involved in the submatrix inversion approach.

However, we can avoid this difficulty by looking at $\rho^{\text {Born }}$ defined in the previous section. Figure $4(\mathrm{~A})$ shows the computed $\rho^{\text {Born }}$ along the complex contour within the
TABLE I: Computed Mülliken populations of gold atoms and cpu times for the full-matrix and submatrix inversion approaches. The energy window of integration is $[-116 \mathrm{eV},-106$ $\mathrm{eV}$, which corresponds to the region of $5 s$ states. The number in parenthesis indicates the position of gold shown in Fig. 1.

\begin{tabular}{lccccc}
\hline \hline Method & $\mathrm{Au}(1)$ & $\mathrm{Au}(2)$ & $\mathrm{Au}(3)$ & $\mathrm{Au}(4)$ & $\mathrm{CPU}$ time (sec) \\
\hline Full-matrix & 1.994 & 1.998 & 1.995 & 1.995 & 414 \\
Submatrix(A) & 1.996 & 1.998 & 1.995 & 1.995 & 243 \\
Submatrix(B) & 1.995 & 1.998 & 1.995 & 1.995 & 245 \\
Submatrix(C) & 1.995 & 1.998 & 1.995 & 1.995 & 248 \\
Submatrix(D) & 1.900 & 1.993 & 1.994 & 1.995 & 251 \\
Submatrix(E) & 1.940 & 1.990 & 1.995 & 1.995 & 259 \\
\hline \hline
\end{tabular}

TABLE II: Computed Mülliken populations of gold atoms and cpu times for the full-matrix and submatrix inversion approaches. The integrated energy window is $[-67 \mathrm{eV},-55 \mathrm{eV}]$, which corresponds to the region of $5 p$ states.

\begin{tabular}{lccccc}
\hline \hline Method & $\mathrm{Au}(1)$ & $\mathrm{Au}(2)$ & $\mathrm{Au}(3)$ & $\mathrm{Au}(4)$ & $\mathrm{CPU}$ time (sec) \\
\hline & & & & & \\
Full-matrix & 5.981 & 5.990 & 5.988 & 5.987 & 436 \\
Submatrix(A) & 5.991 & 5.990 & 5.988 & 5.987 & 260 \\
Submatrix(B) & 5.981 & 5.990 & 5.988 & 5.987 & 264 \\
Submatrix(C) & 5.985 & 5.991 & 5.988 & 5.987 & 269 \\
Submatrix(D) & 5.989 & 5.986 & 5.988 & 5.987 & 291 \\
\hline \hline
\end{tabular}

energy window of $[-116 \mathrm{eV},-106 \mathrm{eV}]$. The values of $\rho^{\text {Born }}$ in submatrix inversions $(\mathrm{A})$ and $(\mathrm{B})$ are quite small at every position on the contour, whereas those in (D) and (E) are quite large, indicating that the divisions of the fullmatrix in (D) and (E) are not good from the viewpoint of the perturbation theory. These behaviors of $\rho^{\text {Born' }}$ 's are consistent with the results in Table I. However, we have an exception, $\operatorname{Submatrix}(\mathrm{C})$, in which $\rho^{\text {Born }}$ is somewhat large, but the computed Mülliken population shows excellent agreement with the full-matrix inversion. Thus, we have to find a threshold for $\rho^{\text {Born }}$ leading to reasonable results. This issue will be studied further in future work.

Table II shows the computed Mülliken populations and cpu times in the energy window of [-67 eV, $-55 \mathrm{eV}]$, which corresponds to the region of the $5 p$ states. Here, we considered four sizes of the submatrix; the matrix sizes (A), (B), (C), and (D) are 128, 158, 190, and 250, respectively. Submatrix (A) includes $5 s$ and $5 p$ states completely, while Submatrices (B), (C), and (D) include valence states located below $-11.0,-9.5$, and $-8.2 \mathrm{eV}$, respectively. In comparison with the full-matrix inversion, the four types of submatrix inversion provide good results. Since the corresponding values of $\rho^{\text {Born }}$ are small, as shown in Fig. 4(B), it can be stated that $\rho^{\text {Born }}$ is a good measure also for the $5 p$ states. The largest difference of the Mülliken population is 0.01 e for $\mathrm{Au}(1)$ in $\operatorname{Submatrix}(\mathrm{A})$. This difference is improved in other submatrices. In addition, computation time is reduced in the submatrix inversion as well as in the case of $5 \mathrm{~s}$ states. Although the threshold of $\rho^{\text {Born }}$ leading to reasonable results must be investigated further, we can conclude that the quantity $\rho^{\text {Born }}$ involves important information which enables us to know the appropriate division into $c$ and $v$ in Eq. (6).

The shielding of the corresponding nuclear charges for 

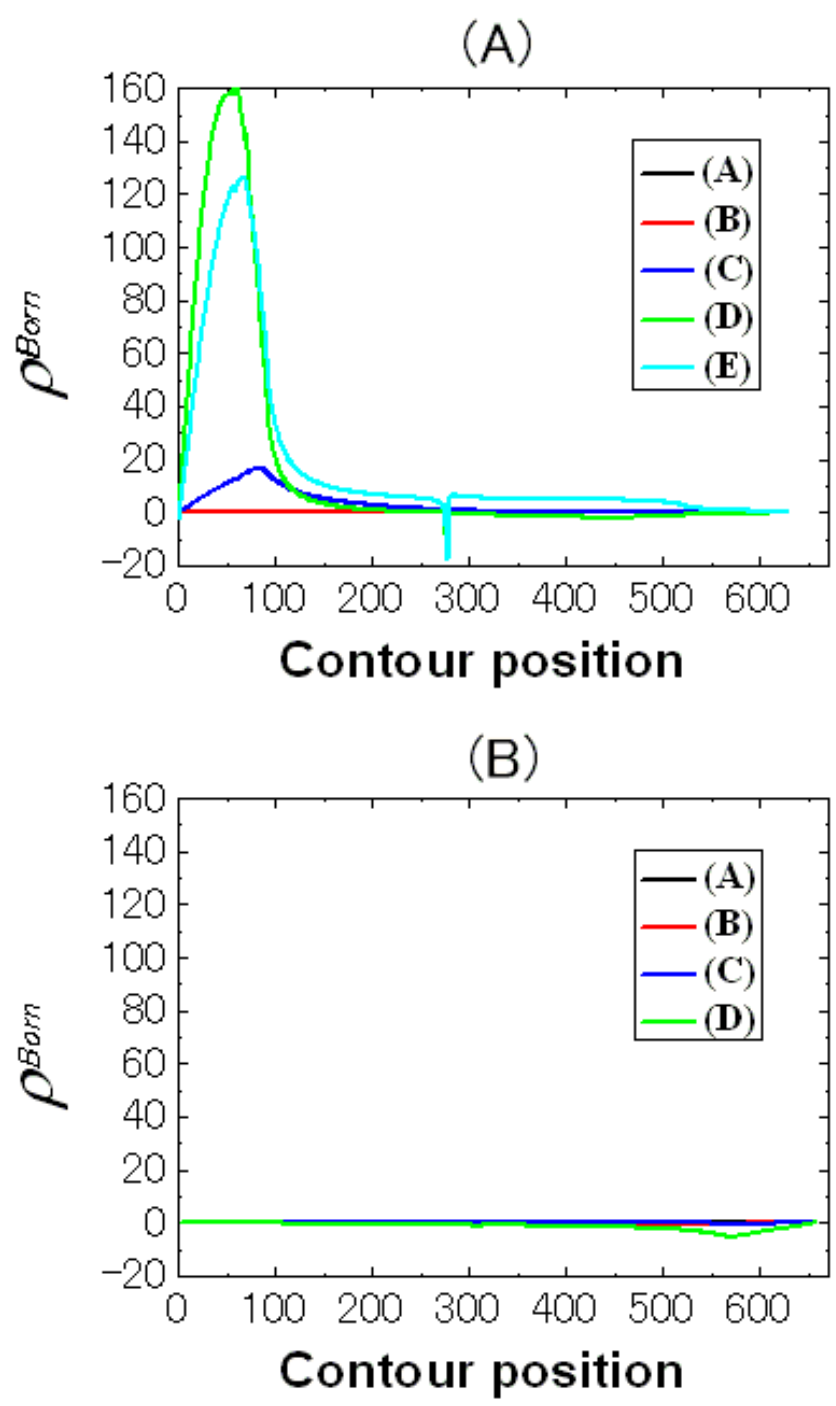

FIG. 4: Computed values of $\rho^{\text {Born }}$ at the mesh points along the contours of (A) $[-116 \mathrm{eV},-106 \mathrm{eV}]$ and $(\mathrm{B})[-67 \mathrm{eV},-55$ $\mathrm{eV}]$.

the $5 s$ and $5 p$ states is almost complete as shown in Tables I and II. Hence, the effects from the core states on transport properties, which are in general dominated by the valence states, will be small in the gold system considered here. However, a system in which the energy interval between the core and valence states is small will show significant contribution of the core states for transport properties. In fact, the modulation of the valence band structure caused by the core states is reported for a perovskite structure that includes $\mathrm{Ti}$ [31]. In addition, the magnetic susceptibility is not adequately described without core states in semiconductors [32], indicating that transport properties of semiconductors under an applied magnetic field must be calculated by including core states.

\section{CONCLUSIONS}

We have proposed a submatrix inversion approach for electrical transport within the framework of the Green's function method incorporated with the GAUSSIAN ab initio program. We have also proposed a Green's function method in terms of molecular orbitals which leads to a reliable contour path in the integration of Green's function. The efficiency and reliability of the proposed approaches were examined for a $\mathrm{Au}_{6}$ linear chain between $\mathrm{Au}(001)$ surfaces, using BPW91 density functional with a modified LANL2MB basis set which supplies $5 s, 5 p$, $5 d, 6 s$, and $6 p$ atomic orbitals for a gold atom. For this model, the integration of Green's function must be performed in the energy window of $\left[-116 \mathrm{eV}, E_{F}\right]$ in conventional approaches, while in the proposed approach, the window is successfully converted into smaller windows of [-116 eV, -106 eV], [-67 eV, -55 eV], and [-15 eV, $\left.E_{F}\right]$. In these windows, we can invert a small submatrix instead of the full-matrix to obtain the whole Green's function. The reduction in computation time by performing the inversion of a small submatrix instead of the full-matrix is about $40 \%$ for both cases of $5 s$ and $5 p$ states. At the same time, we also achieved excellent quantitative agreement of the Mülliken populations with the full-matrix approach. Further, we proposed a new quantity which is useful to determine an appropriate size of the submatrix.

Since conventional approaches for electrical transport do not include core states, this submatrix inversion approach for core states will be a useful tool to investigate the effects of the core states on transport properties. In addition, this approach may also be useful to investigate photocurrent systems induced by the excitation of core electrons.

\section{Acknowledgments}

This study is supported by a Grant-in-Aid for scientific research from the Ministry of Education, Culture, Sports, Science and Technology of Japan.
[1] B. Xu and N. J. Tao, Science 301, 1221 (2003).

[2] S. Csonka, A. Halbritter, and G. Mihály, Phys. Rev. Lett. 90, 116803 (2003).

[3] R. H. M. Smit, C. Untiedt, G. Rubio-Bollinger, R. C. Segers, and J. M. van Ruitenbeek, Phys. Rev. Lett. 91, 076805 (2003).

[4] P. Damle, A. W. Ghosh, and S. Datta, Chem. Phys. 281, $171(2002)$.

[5] E. Louis, J. A. Vergés, J. J. Palacios, A. J. Pérez-Jiménez, and E. SanFabián, Phys. Rev. B 67, 155321 (2003).
[6] Y. J. Lee, M. Brandbyge, M. J. Puska, J. Taylor, K. Stokbro, and R. M. Nieminen, Phys. Rev. B 69, 125409 (2004).

[7] L. de la Vega, A. Martín-Rodero, A. L. Yeyati, and A. Saúl, Phys. Rev. B 70, 113107 (2004).

[8] M. A. Reed, C. Zhou, C. J. Muller, T. P. Burgin, and J. M. Tour, Science 278, 252 (1997).

[9] X. Xiao, B. Xu, and N. J. Tao, Nano Lett. 4, 267 (2004).

[10] M. D. Ventra, S. T. Pantelides, and N. D. Lang, Phys. Rev. Lett. 84, 979 (2000).

[11] K. Stokbro, J. Taylor, M. Brandbyge, J. -L. Mozos, and 
P. Ordejón, Comput. Mater. Sci. 27, 151 (2003).

[12] J. Nara, H. Kino, N. Kobayashi, M. Tsukada, and T. Ohno, Thin Solid Films 438-439, 221 (2003).

[13] F. Evers, F. Weigend, and M. Koentopp, Phys. Rev. B 69, 235411 (2004).

[14] P. Delaney and J. C. Greer, Phys. Rev. Lett. 93, 036805 (2004).

[15] Y. Zhu, J. Maciejko, T. Ji, and H. Guo, Phys. Rev. B 71, 075317 (2005).

[16] A. Ferretti, A. Calzolari, R. D. Felice, F. Manghi, M. J. Caldas, M. B. Nardelli, and E. Molinari, Phys. Rev. Lett. 94, 116802 (2005).

[17] N. Sai, M. Zwolak, G. Vignale, and M. D. Ventra, Phys. Rev. Lett. 94, 186810 (2005).

[18] T. Tada, M. Kondo, and K. Yoshizawa, J. Chem. Phys. 121, 8050 (2004).

[19] T. Tada, S. Hamayama, M. Kondo, and K. Yoshizawa, J. Phys. Chem. B 109, 12443 (2005).

[20] M. Kondo, T. Tada, and K. Yoshizawa, Chem. Phys. Lett. 412, 55 (2005).

[21] S. Tanibayashi, T. Tada, S. Watanabe, and K. Yoshizawa,
Jpn. J. Appl. Phys. 44, 7729 (2005).

[22] M. J. Frisch, et al., Gaussian, Inc., Pittsburgh PA, 2003.

[23] T. H. Dunning Jr. and P. J. Hay, Modern Theoretical Chemistry, Vol. 3 (Plenum, New York, 1976).

[24] W. R. Wadt and P. J. Hay, J. Chem. Phys. 82, 284 (1985).

[25] P. J. Hay and W. R. Wadt, J. Chem. Phys. 82, 299 (1985).

[26] S. Datta, Electronic Transport in Mesoscopic Systems, (Cambridge University Press, Cambridge, 1995).

[27] F. Guinea, C. Tejedor, F. Flores, and E. Louis, Phys. Rev. B 28, 4397 (1983).

[28] A. D. Becke, Phys. Rev. A 38, 3098 (1988).

[29] J. P. Perdew, J. A. Chevary, S. H. Vosko, K. A. Jackson, M. R. Pederson, D. J. Singh, and C. Fiolhais, Phys. Rev. B 46, 6671 (1992).

[30] J. Taylor, H. Guo, and J. Wang, Phys. Rev. B 63, 245407 (2001).

[31] Ph. Ghosez, J. -P. Michenaud, and X. Gonze, Phys. Rev. B 58, 6224 (1998).

[32] K. Ohno, F. Marui, and S. G. Louie, Phys. Rev. B 56, 1009 (1997). 